\title{
Endoscopic skull base reconstruction: a review and clinical case series of 152 vascularized flaps used for surgical skull base defects in the setting of intraoperative cerebrospinal fluid leak
}

\author{
Brian D. Thorp, M.D., ${ }^{1}$ Satyan B. Sreenath, B.S., ${ }^{1}$ Charles S. Ebert, M.D., M.P.H., ${ }^{1}$ \\ and Adam M. Zanation, M.D. ${ }^{1,2}$ \\ Departments of ${ }^{1}$ Otolaryngology-Head and Neck Surgery and ${ }^{2}$ Neurosurgery, The University of North \\ Carolina at Chapel Hill, North Carolina
}

\begin{abstract}
Endoscopic skull base surgery continues to rapidly evolve, requiring comparable advances in reconstructive techniques. While smaller skull base defects with low intraoperative CSF flow have been successfully managed with a variety of avascular and/or noncellular techniques, larger defects with high CSF flow require more robust repairs often in the form of vascularized flaps, which confer excellent success rates in this setting. Despite these successful outcomes, a paucity of data describing specific patient and operative characteristics and their effects on repair exist. Therefore, a retrospective, consecutive chart review was performed on patients who underwent endoscopic skull base reconstruction with a vascularized flap in the setting of intraoperative CSF leaks. In this series, 151 patients with a mean age of 51 years underwent 152 vascularized flap skull base reconstructions for an array of benign and malignant pathologies. These vascularized flaps included 144 nasoseptal flaps, 6 endoscopic-assisted pericranial flaps, 1 facial artery buccinator flap, and 1 inferior turbinate flap that were used throughout all regions of the skull base. Perioperative ( $<3$ months) and postoperative ( $>3$ months) flap complications were assessed and revealed 3 perioperative flap defects $(2.0 \%)$ defined as a visualized defect within the substrate of the flap and a total of 5 perioperative CSF leaks (3.3\%). No patient experienced flap death/complete flap loss in the cohort. Assessed postoperative flap complications included 1 case $(0.7 \%)$ of mucocele formation, 8 cases $(5.3 \%)$ of prolonged skull base crusting, and 2 cases $(1.3 \%)$ of donor-site complication, specifically septal perforation secondary to nasoseptal flap harvest. Among the 152 cases identified, 37 patients received radiation therapy while 114 patients did not undergo radiation therapy as part of the treatment profile. No significant association was found between perioperative complication rates and radiation therapy $(\mathrm{p}=0.634)$. However, a significant association was found between postoperative complication rates and radiation therapy, primarily accounted for by an increased risk for prolonged ( $>6$ months) skull base crusting $(\mathrm{p}=0.025)$. It is clear that larger skull base defects with high intraoperative CSF flow require thoughtful approach and strong consideration for vascularized repair.
\end{abstract}

(http://thejns.org/doi/abs/10.3171/2014.7.FOCUS14350)

\section{KEY WORDS • skull base reconstruction • skull base surgery • vascularized repair}

$\mathrm{O}$ VER the past 15 years, the endoscopic endonasal approach (EEA) has revolutionized the surgical management of sinonasal and skull base pathology. With concurrent advancements in endoscopic instrumentation, radiographic image-based neuronavigation, and the understanding of anatomical relationships, the EEA has expanded to various zones of the ventral skull base for the surgical management of an array of pathology. ${ }^{7}$ As increasingly complex intradural and extradural pathology has been managed via the EEA, the difficulty of associated skull base and dural reconstruction has also significantly increased.

Abbreviations used in this paper: $\mathrm{EEA}=$ endoscopic endonasal approach; FAB = facial artery buccinators flap; ITF = inferior turbinate flap; $\mathrm{NSF}=$ nasoseptal flap; $\mathrm{PCF}=$ pericranial flap; $\mathrm{SNUC}=$ sinonasal undifferentiated carcinoma.
Historically, smaller skull base defects were most commonly repaired using free grafts including mucoperichondrium, mucoperiosteum, and/or fascia. In addition to the aforementioned strategies, free fat grafts and noncellular grafting materials like Alloderm have been commonly used in dural repair.,12 Although these forms of repair have exhibited high success rates in the repair of smaller defects, skull base pathology requiring extensive resection with large resultant dural defects demonstrated the need for more robust forms of repair, which led to the introduction and characterization of pedicled, vascularized flaps.

The advent of pedicled, vascularized skull base repair began in the mid-2000s with the description of the Hadad-Bassagasteguy flap, more commonly known as the nasoseptal flap. ${ }^{2}$ Since this description, many additional 
forms of vascularized repair have been characterized including, but not limited to, the endoscopic-assisted pericranial flap, the tunneled temporoparietal fascia flap, the facial artery buccinator flap, the palatal flap, the middle turbinate flap, and the inferior turbinate flap..$^{9}$ A detailed description of the surgical techniques required for this diverse group of pedicled flaps is outside the scope of this review. However, it is important to note that while the nasoseptal flap remains one of the most commonly used flaps for the skull base reconstructive surgeon, a thorough understanding of secondary options and techniques for vascularized skull base repair is critical.

Given the relatively recent development and expansion of the field of endoscopic skull base surgery and reconstruction, much of the current literature has focused on feasibility and immediate perioperative outcomes. There is a comparative paucity of data assessing longterm outcomes of vascularized repair with an emphasis on operative and/or patient variables and their effect on reconstructive success. Such variables include tumor pathology, defect location, defect size, and utilization of preor postoperative radiation therapy.

The role of radiation therapy on reconstructive success is of particular interest as the scope of EEA has expanded to an array of pathologies in diverse locations. The potential effects of radiation therapy have been noted anecdotally in a multitude of studies and reviews. Moreover, in a review of high-flow CSF leaks repaired with the nasoseptal flap by Zanation et al., a proclivity toward postoperative CSF leak was noted in patients with a history of preoperative radiation therapy. ${ }^{14}$ In addition to the potential effects on reconstructive efforts, radiation therapy also subjects the patient to the risk of treatment complications such as mucositis, wound breakdown and resultant fistula formation, and osteoradionecrosis, all of which can be further compounded by patient factors. ${ }^{6}$

Thus, the primary objective of this study is to investigate and characterize the long-term outcomes of vascularized skull base reconstruction at our institution. These outcomes are stratified by different patient and operative factors, allowing a more thorough understanding of the effect of these variables on vascularized repair in the endoscopic era.

\section{Methods}

Institutional review board approval was obtained to perform a retrospective, consecutive chart review at our institution. Patients who underwent endoscopic skull base reconstruction with a vascularized flap in the setting of intraoperative CSF leak by the senior authors (A.M.Z. and C.S.E.) who had long-term clinical and radiographic follow-up were identified and included. Patients were excluded if the primary operation involved an open surgical approach. Medical records were reviewed for patient demographic information, pathology, characteristics of approach, presence and dosage of pre- or postoperative radiation therapy, type of vascularized flap used, flap complications, perioperative CSF leak, and other major patient complications. Statistical significance was assessed within our data using Fisher's exact tests.

\section{Results}

\section{Patient Characteristics}

A retrospective review yielded 151 patients who underwent a total of 152 vascularized flap skull base reconstructions in the setting of an intraoperative CSF leak. The average age of our cohort was 51 years, with 64 male patients and 87 female patients identified. Additional demographic information is detailed in Table 1. Of the 151 patients identified, 126 patients exhibited a wide range of benign pathology while the remaining 25 patients displayed a variety of malignant pathology. These diverse pathological characteristics are detailed in Table 2. Evaluation of the type of skull base reconstructive technique used revealed 144 patients who underwent reconstruction with the nasoseptal flap (NSF), 6 patients with an endoscopic-assisted pericranial flap (PCF), 1 patient with a facial artery buccinator flap (FAB), and 1 patient with an inferior turbinate flap (ITF). Further analysis revealed 32 patients who underwent postoperative radiation therapy, 5 patients who underwent preoperative radiation therapy, and 114 patients who did not receive any radiation therapy as part of their treatment profile. The average clinical and radiographic follow-up time for all patients was 13 months, with a median of 8 months and a range of 1-38 months.

\section{Flap Characteristics and Complications}

The most common indication for vascularized skull base reconstruction in the studied cohort was for the repair of a sellar defect in the setting of intraoperative CSF leak encountered during endoscopic endonasal transsphenoidal resection of a pituitary adenoma (52 [34.2\%] of 152). The NSF was used for an array of skull base defects in the cohort including, most commonly, sellar reconstruction $(52$ [36.1\%] of 144), followed by anterior skull base reconstruction (87 [60.4\%] of 144) of the following regions: frontal, frontotemporal, cribriform, ethmoid,

\section{TABLE 1: Study population and associated demographics*}

\begin{tabular}{lc}
\hline Population/Demographic & Value \\
\hline total no. of flaps & 152 \\
no. of patients & 151 \\
male & 64 \\
female & 87 \\
mean age in yrs & 50.6 \\
mean follow-up in mos & 13 \\
mean hospital stay in days & 6.4 \\
common comorbidities & \\
hypertension & $76(50.3)$ \\
diabetes mellitus & $23(15.2)$ \\
dyslipidemia & $15(9.9)$ \\
obstructive sleep apnea & $13(8.6)$ \\
smoking history & $10(6.6)$ \\
asthma/COPD & $8(5.3)$ \\
\hline
\end{tabular}

* Values are number of patients (\%) unless stated otherwise. COPD = chronic obstructive pulmonary disease. 


\section{Endoscopic skull base reconstruction}

TABLE 2: Benign and malignant skull base pathology*

\begin{tabular}{lc}
\hline \multicolumn{1}{c}{ Skull Base Pathology } & $\begin{array}{c}\text { No. of Patients } \\
\text { (\% subgroup) }\end{array}$ \\
\hline benign & 126 \\
pituitary adenoma & $58(46.0)$ \\
encephalocele/CSF leak & $34(27.0)$ \\
meningioma & $13(10.3)$ \\
craniopharyngioma & $10(7.9)$ \\
dermoid/epidermoid & $2(1.6)$ \\
chordoma & $2(1.6)$ \\
granular cell tumor & $1(0.8)$ \\
epidermoid cyst & $1(0.8)$ \\
hemangioma & $1(0.8)$ \\
osteoradionecrosis & $1(0.8)$ \\
Rathke's cleft cyst & $1(0.8)$ \\
osteoma & $1(0.8)$ \\
spindle cell oncocytoma & $1(0.8)$ \\
malignant & 25 \\
esthesioneuroblastoma & $5(20.0)$ \\
SNUC & $4(16.0)$ \\
squamous cell carcinoma of skull base & $4(16.0)$ \\
mucosal melanoma & $4(16.0)$ \\
immature teratoma & $2(8.0)$ \\
basaloid cell carcinoma & $1(4.0)$ \\
B-cell lymphoma & $1(4.0)$ \\
juvenile pilocytic astrocytoma & $1(4.0)$ \\
malignant peripheral nerve sheath tumor & $1(4.0)$ \\
adenocarcinoma of skull base & $1(4.0)$ \\
squamous cell carcinoma arising from IP & $1(4.0)$ \\
\hline
\end{tabular}

* $\mathrm{IP}=$ inverted papilloma.

planum sphenoidale, and lateral sphenoid. Vascularized reconstruction of the clivus (5 [3.5\%] of 144) with the NSF was also noted. The PCF was exclusively used for endoscopic reconstruction of larger defects of the anterior skull base. Lastly, the FAB and ITF were used as secondary options in patients in whom the NSF had either been previously harvested or was unusable for primary reconstruction. It is important to emphasize that only patients with intraoperative CSF leaks were included in the study.

Given the large number of NSFs used for sellar reconstruction in the cohort, it is important to review the indications and technique for this application. After preparing the bilateral nasal passages for EEA, the middle turbinate is resected. This is most commonly performed on the right unless exposure secondary to septal deviation or other sinonasal abnormality limits visualization. At this point, a nasoseptal rescue flap is developed as described by Rivera-Serrano et al. ${ }^{11}$ Briefly, an incision beginning at the sphenoid os is carried across the sphenoid rostrum to the posterior, superior osseous septum where it is swept superiorly and then anteriorly for approximately one-third to one-half of the superior septum along the sagittal plane. Once incised, a submucoperiosteal/sub- mucoperichondrial plane is developed, allowing the mucosa to be freed beyond the level of the sphenoid floor or posterior choanae, thereby protecting and preserving the posterior septal artery during further dissection. A posterior septectomy including the contralateral mucosa is then performed, allowing wide bilateral sphenoid opening and sellar exposure. If an intraoperative CSF leak is not encountered, the rescue flap is simply repositioned. However, if an intraoperative CSF leak is noted, the rescue flap is then converted to a standard nasoseptal flap and used for skull base reconstruction. ${ }^{11}$ Importantly, this technique is only used for sellar tumors with minimal parasellar and suprasellar extension, and a standard nasoseptal flap is raised initially for all other cases not meeting these criteria.

In this series of 152 vascularized flap reconstructions, flap complications were categorized into perioperative ( $<3$ months) and postoperative ( $>3$ months). Perioperative flap complications assessed include flap death defined as complete flap loss, flap defect defined as a visualized defect within the substrate of the flap, and CSF leak. Postoperative flap complications assessed include CSF leak, encephalocele formation, mucocele formation, prolonged skull base crusting as appreciated on routine postoperative sinonasal endoscopy, and donor site complications. Of the identified perioperative complications, 3 flap defects $(2.0 \%)$ and a total of 5 CSF leaks (3.3\%) were identified, including those resulting from the noted flap defect. No patient experienced flap death/complete flap loss in the cohort. Assessed postoperative flap complications included 1 case $(0.7 \%)$ of mucocele formation, 8 cases $(5.3 \%)$ of prolonged skull base crusting, and 2 cases $(1.3 \%)$ of donor-site complication, specifically septal perforation secondary to NSF harvest. While not specifically detailed, in 122 (84.7\%) of the 144 nasoseptal flaps studied in this cohort, a middle turbinate autograft was placed at the donor site. In these cases, significantly reduced donor site complications $(\mathrm{p}=0.018)$ were found. No postoperative ( $>3$ months) CSF leaks or secondary encephalocele formations were noted. These findings are summarized in Table 3.

Perioperative and postoperative flap complications were also stratified by the inclusion of radiation therapy to the treatment profile. Among the 151 patients identified, 32 received adjuvant postoperative radiation therapy, 5 received neoadjuvant preoperative radiation therapy, and 114 did not undergo radiation therapy. Of the patients whose cumulative radiation dose was known, the average dose was 56.7 Gy. No significant association was found between perioperative complication rates and radiation therapy $(\mathrm{p}=0.634)$. However, a significant association was found between postoperative complication rates and radiation therapy, primarily accounted for by an increased risk for prolonged ( $>6$ months) skull base crusting $(\mathrm{p}=0.025)$.

Perioperative and postoperative complications were also stratified by the location of the resultant skull base defect, which were categorized into the following subsites: anterior skull base, sella, and clivus (Table 4). The anterior skull base incorporated defects of the frontal, frontotemporal, cribriform, ethmoid, planum sphenoi- 
TABLE 3: Perioperative and postoperative flap complications

\begin{tabular}{cc}
\hline \multicolumn{1}{c}{ Complications } & Total (\% total flaps) \\
\hline periop (<3 mos) & \\
CSF leak & $5(3.3)$ \\
flap defect & $3(2.0)$ \\
flap death & $0(0.0)$ \\
postop (>3 mos) & \\
prolonged skull base crusting & $8(5.3)$ \\
donor site complications & $2(1.3)$ \\
mucocele formation & $1(0.7)$ \\
CSF leak & $0(0.0)$ \\
secondary encephalocele & $0(0.0)$ \\
\hline
\end{tabular}

dale, and lateral sphenoid regions. An increase in perioperative or postoperative complication risk was found when comparing anterior skull base defects and sellar defects $(p=0.003)$. However, this increase in complication risk was not seen when comparing anterior skull base defects and clival defects $(\mathrm{p}=0.0721)$ and sellar defects and clival defects $(\mathrm{p}=0.087)$.

\section{Radiation Status and Complication Profile}

Of the 37 patients who received either adjuvant or neoadjuvant radiation therapy, 24 patients exhibited malignant pathology, and 13 patients demonstrated benign pathology with aggressive characteristics. Of the 114 patients who did not receive radiation therapy, there were 3 cases $(2.6 \%)$ of meningitis, 3 cases $(2.6 \%)$ of CSF leak, 2 cases $(1.8 \%)$ of pneumocephalus, and 2 cases $(1.8 \%)$ of prolonged skull base crusting. In the 37 patients who received radiation therapy, there were 6 cases $(16.2 \%)$ of prolonged skull base crusting, 4 cases $(10.8 \%)$ of osteoradionecrosis, 3 cases $(8.1 \%)$ of CSF leak, 3 cases $(8.1 \%)$ of pneumocephalus, 3 cases $(8.1 \%)$ of nasal vestibular stenosis, 1 case $(2.7 \%)$ of meningitis, and 1 case $(2.7 \%)$ of frontal cerebritis (Table 5). Specific evaluation of the role of radiation therapy and CSF leak revealed no significant difference in the rates of CSF leak between those receiving radiation therapy and those not receiving radiation therapy $(p=0.464)$. Interestingly, a newly identified skull base CSF leak was identified distal from the initial reconstruction site in a single patient. This site was in the region of locally recurrent mucosal melanoma and was thought to result from a combination of treatment effect and disease progression. This was not considered a reconstruction failure and thus was not included with those CSF leaks occurring at the site of skull base defect repair.

\section{CSF Leakage}

All cases (5 [3.3\%]) of CSF leak requiring secondary repair in our cohort are outlined in Table 6. Included in this small cohort of vascularized flap failures are 3 NSFs, $1 \mathrm{PCF}$, and $1 \mathrm{ITF}$. Benign pathology that did not require radiation therapy was noted in 4 of the 5 flap failures. It should also be noted that 2 of the 5 cases of flap failure were seen in the same patient, who underwent resection of a large epidermoid tumor requiring reconstruction of an extensive clival defect. All cases of CSF leaks were classified as high-flow CSF leaks intraoperatively.

The average duration from original intraoperative repair to CSF leak was 43.6 days. It should be noted that this average may be skewed by a single delayed failure that occurred 90 days after initial repair in the setting of a large anterior skull base defect following radiation therapy for the management of sinonasal undifferentiated carcinoma (SNUC). Removing this patient results in an average duration from original repair to CSF leak of 32 days. The majority of secondary skull base repairs (3/5) were managed using a multilayered closure involving readjustment of the original flap and bolstering with synthetic, noncellular material. The remaining secondary repairs were managed as follows: 1 defect was reconstructed using a new vascularized flap and 1 was repaired using a combination of avascular and noncellular grafts, including abdominal fat and Durepair. All CSF leaks were managed with a single secondary repair with the exception of one that required a tertiary repair. Lumbar drainage was not routinely used in the primary setting and was often reserved for secondary repair in the setting of initial reconstructive failure. A lumbar drain was only used in $60 \%$ of those patients undergoing secondary repair.

\section{Discussion}

The increasing complexity of pathology addressed with the EEA has resulted in advancements in the strategies for skull base reconstruction. Through this evolution, a multitude of techniques utilizing free avascular grafts, free synthetic noncellular grafts, and/or pedicled, vascular flaps have been described. While overall postoperative CSF leak rates, regardless of reconstructive technique, have approached $8.5 \%$ in recent reviews, signifi-

TABLE 4: Flap complications stratified by defect location

\begin{tabular}{lcccc}
\hline Skull Base Defect Location & $\begin{array}{c}\text { No. of Cases w/ Any Flap } \\
\text { Complication (\% subgroup) }\end{array}$ & $\begin{array}{c}\text { No. of Cases w/o Flap } \\
\text { Complication (\% subgroup) }\end{array}$ & $\begin{array}{c}\text { Total No. of Cases/ } \\
\text { Defect Location }\end{array}$ & p Value \\
\hline anterior skull base & $13(22.4)$ & $45(77.6)$ & 58 & 0.003 \\
sella & $1(1.5)$ & $65(98.5)$ & 66 & 0.721 \\
anterior skull base & $13(22.4)$ & $45(77.6)$ & 58 & 0.087 \\
clivus & $2(13.3)$ & $13(86.7)$ & 66 & \\
sella & $1(1.5)$ & $65(98.5)$ & 15 & \\
clivus & $2(13.3)$ & $13(86.7)$ & & \\
\hline
\end{tabular}


TABLE 5: Total patient complications stratified by radiation status

\begin{tabular}{lc}
\hline \multicolumn{1}{c}{ Complication } & $\begin{array}{c}\text { No. of Patients } \\
\text { (\% subgroup) }\end{array}$ \\
\hline $\begin{array}{c}\text { radiation therapy }(\mathrm{n}=37) \\
\text { prolonged skull base crusting }\end{array}$ & $6(16.2)$ \\
osteoradionecrosis & $4(10.8)$ \\
CSF leak & $3(8.1)$ \\
pneumocephalus & $3(8.1)$ \\
nasal vestibular stenosis & $3(8.1)$ \\
frontal cerebritis & $1(2.7)$ \\
meningitis & $1(2.7)$ \\
no radiation therapy ( $=114)$ & \\
meningitis & $3(2.6)$ \\
CSF leak & $3(2.6)$ \\
pneumocephalus & $2(1.8)$ \\
prolonged skull base crusting & $2(1.8)$ \\
\hline
\end{tabular}

cant emphasis has been placed on the role of defect size, defect location, and degree of intraoperative CSF leak. ${ }^{13}$ In fact, when these factors are used for data stratification, it is clear that vascularized repair is superior to avascular techniques. ${ }^{3,13}$

Harvey et al. and Soudry et al. performed large systematic reviews of the literature, assessing endoscopic skull base reconstruction. ${ }^{3,13}$ In the review of 38 studies by Harvey et al., 609 patients with large dural defects were identified. From this cohort, 326 underwent free graft reconstruction while 283 underwent vascularized reconstruction, resulting in a statistically significant difference in the rate of CSF leak of $15.6 \%$ (51 of 326) and $6.7 \%$ (19 of 283) for free graft and vascularized reconstruction, respectively. ${ }^{3}$ In the review of 22 studies by Soudry et al., an overall postoperative CSF leak rate after skull base repair of $8.5 \%$ (57 of 673) was found, revealing an overall success of $91.5 \%$. To further this evaluation, the authors focused additional inquiry on the role of the location of skull base defect and degree of intraoperative CSF leak. Utilizing these criteria, no difference between nonvascularized and vascularized reconstruction was appreciated for any location subsite except the clivus, which demonstrated better reconstructive results with vascularized repair. Consideration of the role of intraoperative CSF leak revealed $92 \%$ success rates with free grafts/synthetic materials with low flow rates. However, cases with high flow rates revealed improved outcomes with vascularized repairs as leak rates of $82 \%$ and $94 \%$ were found with free grafts/synthetic materials and vascularized repairs, respectively. ${ }^{13}$

The immense versatility of the NSF and the ability to cover a reconstructive area averaging $17.12 \mathrm{~cm}^{2}$ as delineated by anatomical and radiographic studies has led to its broad use in skull base reconstruction. ${ }^{10}$ Moreover, CSF leak rates of $5.7 \%$ when used for skull base repair in the setting of high intraoperative CSF flow have been reported. ${ }^{14}$ While the NSF remains the primary option for vascular skull base repair, other options demonstrate comparable success rates and represent excellent options for the experienced surgeon. ${ }^{9}$ While describing many of these secondary options for skull base repair, Patel et al. found that these repairs conferred a postoperative CSF leak rate of $3.6 \%$, a finding comparable to the success rate of the nasoseptal flap. ${ }^{8}$

While it remains clear that the surgeon must consider a multitude of factors when choosing a strategy for reconstruction, several patient and treatment factors exist that may further augment the treatment algorithm. As radiation therapy represents a significant component of the treatment of complex skull base pathology, its perioperative and postoperative effects have been implicated in reconstructive failure risk. ${ }^{14}$ In our cohort, no significant difference was seen in patients receiving radiation therapy and those not receiving radiation therapy $(p=0.464)$. Only a single patient who received radiation therapy (1 [2.7\%] of 37) was noted to have a CSF leak. This leak occurred following a course of chemoradiotherapy. The patient's initial defect was large and occupied the anterior skull base with high intraoperative CSF flow. This was managed with flap repositioning and a combination of a free abdominal fat graft and synthetic graft. While radiation therapy did not impart a significant effect on reconstructive success in our cohort, it remains an important consideration when selecting a technique for repair. Additionally, only vascularized flaps were used within the study population and subgroup analysis of avascular techniques, if available, may have resulted in significance when comparing these modalities. When assessing the literature for comparable evaluations of the effect of radiation therapy, a study was identified assessing the effect of adjuvant radiotherapy on changes in a thigh free flap reconstruction following total parotidectomy. A mean volume loss of $8 \%$ of the free flap was noted 6 months

\section{TABLE 6: Cerebrospinal fluid leaks}

\begin{tabular}{|c|c|c|c|c|c|c|c|}
\hline $\begin{array}{l}\text { Leak } \\
\text { No. }\end{array}$ & Pathology & Defect Location & $\begin{array}{l}\text { Time Until CSF Leak } \\
\text { Identified (days) }\end{array}$ & $\begin{array}{l}\text { Type of Initial } \\
\text { Reconstruction }\end{array}$ & $\begin{array}{l}\text { Type of Secondary/ } \\
\text { Revision Repair }\end{array}$ & Radiotherapy & $\begin{array}{c}\text { Intraop } \\
\text { Lumbar Drain }\end{array}$ \\
\hline 1 & encephalocele & anterior skull base & 12 & NSF & NSF/Alloderm & no & no \\
\hline 2 & epidermoid tumor & clivus & 60 & NSF & ITF & no & yes \\
\hline 3 & epidermoid tumor & clivus & 40 & ITF & ITF/Alloderm & no & yes \\
\hline 4 & craniopharyngioma & planum/sella & 16 & NSF & $\begin{array}{l}\text { NSF/Alloderm/abdomi- } \\
\text { nal fat }\end{array}$ & no & no \\
\hline 5 & SNUC & anterior skull base & 90 & PCF & Durepair/abdominal fat & yes & yes \\
\hline
\end{tabular}


after radiation thereapy. ${ }^{5}$ In a larger study investigating the impact of preoperative radiotherapy on microvascular free flap reconstruction, it was noted that preoperative radiation $\geq 60$ Gy was associated with increased risk of free flap failure and overall local complications. ${ }^{1}$

While significant focus is placed on reconstructive success, patients receiving radiation therapy are also susceptible to additional complications. These potential complications include prolonged skull base crusting, osteoradionecrosis, pneumocephalus, nasal vestibular stenosis, meningitis, and frontal cerebritis and require careful acute and long-term follow-up.

An overall CSF leakage rate of $3.3 \%$ was found within the patient cohort. All of these leaks occurred in the perioperative period ( $<3$ months) and included 3 NSFs, $1 \mathrm{PCF}$, and 1 ITF. As previously noted, radiation therapy status did not result in a significant increase in risk for flap failure. Additionally, defect location did not confer a significant increase in risk for flap failure. However, as detailed in Table 3 and synthesized in Table 4, a comparison of the anterior skull base and sella revealed a significant increase in the risk of any complication between these sites. This significance was largely driven by prolonged skull base crusting rather than more significant complications like CSF leakage.

When further assessing the CSF leaks identified in this cohort, 3 of the 5 failures resulted from flap defects appreciated at the time of secondary repair. These flap defects were small, pinpoint fistulas within the substrate of the flap. They were invariably located at dependent positions where underlying CSF was able to pool and impart additional pressure on the reconstruction. Importantly, these fistulas were not the result of flap harvest as none of the included vascularized flaps demonstrated abnormality at the time of elevation. The remaining flap failures occurred at the periphery of the reconstruction. These were not thought to be secondary to intraoperative flap positioning as complete defect coverage was noted intraoperatively in both cases. Moreover, as detailed above, one of these failures occurred following a course of chemoradiotherapy for a large anterior skull base SNUC with large resultant defect and high intraoperative CSF flow.

Finally, of the 5 reported leaks, 2 occurred in a single patient with a complex clival defect. Neither of these leaks occurred acutely and when each was visually identified they were in the milieu of extensive skull base crusting. At the time of secondary repair, a peripheral leak was noted and managed with an ITF. At the time of tertiary repair, a pinpoint fistula was appreciated and repaired with placement of an underlay synthetic graft. Thus, it is clear that clival defects with large posterior fossa dural openings remain a challenging reconstructive endeavor and require a thoughtful approach with strong consideration for a vascularized repair.

Endoscopic skull base surgery continues to rapidly evolve with ever-increasing indications for the management of challenging extradural and intradural pathology. This rapid evolution has required comparable advances in reconstructive techniques. Vascularized repair results in excellent success rates, especially in the setting of large defects with high CSF flow. Given the high success rates of these vascular techniques, other factors remain important considerations in the reconstructive algorithm but are challenging to statistically evaluate. Despite a lack of significance in this study, the role of radiation therapy requires strong reconstructive consideration especially when compounded by larger defects with high intraoperative CSF flow.

\section{Conclusions}

The current study and review of the literature strongly supports the utilization of vascularized repairs for skull base defects given a success rate of greater than $95 \%$. Beyond the technical considerations, careful preoperative and intraoperative planning are required to ensure selection of the appropriate reconstructive method. Additionally, one must account for the potential patient and treatment effects that may augment a technically successful repair. Continued inquiry will further our understanding of risk stratification and allow for additional improvements in this rapidly expanding field.

\section{Disclosure}

The authors report no conflict of interest concerning the materials or methods used in this study or the findings specified in this paper.

Author contributions to the study and manuscript preparation include the following. Conception and design: all authors. Acquisition of data: all authors. Analysis and interpretation of data: all authors. Drafting the article: all authors. Critically revising the article: all authors. Reviewed submitted version of manuscript: all authors. Approved the final version of the manuscript on behalf of all authors: Thorp. Statistical analysis: all authors.

\section{References}

1. Benatar MJ, Dassonville O, Chamorey E, Poissonnet G, Ettaiche M, Pierre CS, et al: Impact of preoperative radiotherapy on head and neck free flap reconstruction: a report on 429 cases. J Plast Reconstr Aesthet Surg 66:478-482, 2013

2. Hadad G, Bassagasteguy L, Carrau RL, Mataza JC, Kassam A, Snyderman $\mathrm{CH}$, et al: A novel reconstructive technique after endoscopic expanded endonasal approaches: vascular pedicle nasoseptal flap. Laryngoscope 116:1882-1886, 2006

3. Harvey RJ, Parmar P, Sacks R, Zanation AM: Endoscopic skull base reconstruction of large dural defects: a systematic review of published evidence. Laryngoscope 122:452-459, 2012

4. Hegazy HM, Carrau RL, Snyderman CH, Kassam A, Zweig $\mathrm{J}$ : Transnasal endoscopic repair of cerebrospinal fluid rhinorrhea: a meta-analysis. Laryngoscope 110:1166-1172, 2000

5. Higgins KM, Erovic BM, Ravi A, Yeung R, Lee JW, Yao C, et al: Volumetric changes of the anterolateral thigh free flap following adjuvant radiotherapy in total parotidectomy reconstruction. Laryngoscope 122:767-772, 2012

6. Jang JW, Chan AW: Prevention and management of complications after radiotherapy for skull base tumors: a multidisciplinary approach. Adv Otorhinolaryngol 74:163-173, 2013

7. Kassam AB, Gardner P, Snyderman C, Mintz A, Carrau R: Expanded endonasal approach: fully endoscopic, completely transnasal approach to the middle third of the clivus, petrous bone, middle cranial fossa, and infratemporal fossa. Neurosurg Focus 19(1):E6, 2005

8. Patel MR, Stadler ME, Snyderman CH, Carrau RL, Kassam AB, Germanwala AV, et al: How to choose? Endoscopic skull base reconstructive options and limitations. Skull Base 20:397-404, 2010 


\section{Endoscopic skull base reconstruction}

9. Patel MR, Taylor RJ, Hackman TG, Germanwala AV, SasakiAdams D, Ewend MG, et al: Beyond the nasoseptal flap: outcomes and pearls with secondary flaps in endoscopic endonasal skull base reconstruction. Laryngoscope 124:846-852, 2014

10. Pinheiro-Neto CD, Ramos HF, Peris-Celda M, FernandezMiranda JC, Gardner PA, Snyderman CH, et al: Study of the nasoseptal flap for endoscopic anterior cranial base reconstruction. Laryngoscope 121:2514-2520, 2011

11. Rivera-Serrano CM, Snyderman CH, Gardner P, Prevedello D, Wheless S, Kassam AB, et al: Nasoseptal "rescue" flap: a novel modification of the nasoseptal flap technique for pituitary surgery. Laryngoscope 121:990-993, 2011

12. Senior BA, Jafri K, Benninger M: Safety and efficacy of endoscopic repair of CSF leaks and encephaloceles: a survey of the members of the American Rhinologic Society. Am J Rhinol 15:21-25, 2001

13. Soudry E, Turner JH, Nayak JV, Hwang PH: Endoscopic reconstruction of surgically created skull base defects: a sys- tematic review. Otolaryngol Head Neck Surg 150:730-738, 2014

14. Zanation AM, Carrau RL, Snyderman CH, Germanwala AV, Gardner PA, Prevedello DM, et al: Nasoseptal flap reconstruction of high flow intraoperative cerebral spinal fluid leaks during endoscopic skull base surgery. Am J Rhinol Allergy 23:518-521, 2009

Manuscript submitted June 15, 2014.

Accepted July 11, 2014.

Please include this information when citing this paper: DOI: 10.3171/2014.7.FOCUS14350.

Address correspondence to: Brian D. Thorp, M.D., Department of Otolaryngology - Head \& Neck Surgery, University of North Carolina at Chapel Hill, 170 Manning Dr., CB \#7070, Physician's Office Building Rm. G-190, Chapel Hill, NC 27599. email: brian_thorp@ med.unc.edu. 